\title{
LBSizeCleav: improved support vector machine (SVM)-based prediction of Dicer cleavage sites using loop/bulge length
}

\author{
Yu Bao*, Morihiro Hayashida and Tatsuya Akutsu
}

\begin{abstract}
Background: Dicer is necessary for the process of mature microRNA (miRNA) formation because the Dicer enzyme cleaves pre-miRNA correctly to generate miRNA with correct seed regions. Nonetheless, the mechanism underlying the selection of a Dicer cleavage site is still not fully understood. To date, several studies have been conducted to solve this problem, for example, a recent discovery indicates that the loop/bulge structure plays a central role in the selection of Dicer cleavage sites. In accordance with this breakthrough, a support vector machine (SVM)-based method called PHDCleav was developed to predict Dicer cleavage sites which outperforms other methods based on random forest and naive Bayes. PHDCleav, however, tests only whether a position in the shift window belongs to a loop/bulge structure.
\end{abstract}

Result: In this paper, we used the length of loop/bulge structures (in addition to their presence or absence) to develop an improved method, LBSizeCleav, for predicting Dicer cleavage sites. To evaluate our method, we used 810 empirically validated sequences of human pre-miRNAs and performed fivefold cross-validation. In both 5p and 3p arms of pre-miRNAs, LBSizeCleav showed greater prediction accuracy than PHDCleav did. This result suggests that the length of loop/bulge structures is useful for prediction of Dicer cleavage sites.

Conclusion: We developed a novel algorithm for feature space mapping based on the length of a loop/bulge for predicting Dicer cleavage sites. The better performance of our method indicates the usefulness of the length of loop/bulge structures for such predictions.

Keywords: Dicer cleavage site, Support vector machine, Loop/bulge length

\section{Background}

MicroRNAs (miRNAs) are a type of small RNAs with the length $\sim 22 \mathrm{nt}$, which perform the function of suppressing gene expression at the post-transcriptional level $[1,2]$. Usually in vivo, a gene of a miRNA is transcribed to produce a long, primary miRNA (pri-miRNA) transcript, which is then processed into a $\sim 65$-nt-long hairpin structure via cleavage by the Drosha (DGCR8) enzyme. Then, the resulting pre-miRNA is cleaved by another enzyme (termed Dicer) to generate a mature miRNA, which is $\sim 22$ nt long [3]. Finally, the generated miRNA can be combined with an Argonaute protein to form the protein-miRNA

*Correspondence: houu@kuicr.kyoto-u.ac.jp Laboratory of Mathematical Bioinformatics, Bioinformatics Center, Institute for Chemical Research, Kyoto University, Gokasho, Uji, 611-0011 Kyoto, Japan complex, which can control various cellular progresses including development, cell death, and metabolism [4-6].

Dicer is a 1922-amino acid multidomain protein that belongs to the RNase III family. Dicer generally contains several domains including ATPase-helicase, DUF283 (a double-stranded-RNA-binding domain), PAZ (PiwiArgonaute-Zwille) domain, two RNase III domains, and a dsRBD [7]. Dicer in various species may contain a different combination of these domains. Among these domains, the PAZ domain, RNase III domain, and dsRND are responsible for the function of substrate cleavage [8]. The cleavage occurs near the end of the terminal loop of pre-miRNA, introducing a cut into the hairpin.

Structural analysis of human Dicer revealed that the PAZ domain contains a $5 \mathrm{p}$ phosphate-binding pocket, which may be necessary for selection of a Dicer cleavage 
site [9]. There are also studies showing that the loop/bulge structure also determines the accuracy of cleavage activity $[10,11]$. MacRae et al. reported that the 3p-terminal nucleotide of single-stranded RNA can affect Dicer binding [12]. In addition, Jin and Lee found that a single nucleotide polymorphism may be associated with miRNA regulation [13]. All these studies revealed that secondary structures of both the Dicer enzyme and cleavage substrates are essential for cleavage site determination.

With a better understanding of the features of selection of a Dicer cleavage site, researchers may be able to elucidate the mechanism of action of enzymes in the RNA III family as well as the processes of RNA inference. Thus, it is imperative to explore the factors affecting the accuracy of Dicer cleavage to gain better insights into the mechanism of Dicer cleavage. Recently, a support vector machine (SVM)-based method (PHDCleav) was developed to predict selection of Dicer cleavage sites [14]. They proposed feature space mappings from premiRNA nucleotide sequences on the basis of existence of a predicted loop/bulge structure. SVM is a state-of-the-art machine learning technology [15] that has been applied to various areas of pattern recognition in many biological fields such as protein classification [16-18], prediction of RNA secondary structure $[19,20]$, and drug-nondrug classification $[21,22]$.

In this paper, we made use of the length of loop/bulge structures and proposed a novel algorithm of feature space mapping called LBSizeCleav. To evaluate our method, we used 810 empirically valid sequences of pre-miRNAs from miRBase and performed fivefold cross-validation. In the $5 \mathrm{p}$ arm of pre-miRNAs, the proposed method attained higher accuracy $(87.4 \%)$, whereas the best prediction result of PHDCleav corresponded to the accuracy of $84.0 \%$ (an extended binary pattern, a window of 14-nt size). In addition, in the $3 \mathrm{p}$ arm, the average prediction accuracy of our method reached $83.0 \%$, whereas PHDCleav achieved up to $79.1 \%$ prediction accuracy. These results suggest that our method LBSizeCleav outperforms binary patterns of PHDCleav in predicting the position of Dicer cleavage sites. The better performance may in turn serve as the evidence that the features utilized by these two methods are necessary for Dicer cleavage selection.

\section{Methods}

In this section, we provide a brief description of feature space mapping algorithms of PHDCleav using sequences and secondary structures and propose a novel algorithm for feature space mapping, LBSizeCleav, based on the length of a loop/bulge structure.

\section{Feature space mapping procedures of PHDCleav}

Given a pre-miRNA sequence, a site between two successive nucleotides is mapped to a binary vector. In
PHDCleav, a window is generated for each input sequence where for the positive pattern the center of the window is exactly located at the cleavage site of $5 p(3 p)$ arm and for the negative pattern the center of the window is located 6 nt away from the cleavage site of $5 p(3 p)$ arm. Since this is based on the assumption that a cleavage site can shift slightly (1-2 nt in biological experiments) but the chance is rare that Dicer cuts in the middle of mature miRNA, 6 nt could be changed under the principle that the center of the negative pattern is far enough from the real cleavage site. PHDCleav has shown that there is little affect to the accuracy of prediction even with the shifting of negative windows among the whole sequence of pre-miRNA.

A nucleotide in a window having the site at the center is converted to a four-dimensional vector as $[1,0,0,0]$, $[0,1,0,0],[0,0,1,0]$, and $[0,0,0,1]$, for A, U, C, and $\mathrm{G}$, respectively (see Table 1 ). Let $w$ denote the size of the window, where $w$ is a positive even number. Then, a $4 w$ dimensional vector is generated for the site.

There are many loops/bulges in the secondary structure of pre-miRNA where one arm contains extra nucleotides without counterparts in the other arm [23]. A recent study indicated that these loops/bulges play an important role in the selection of a Dicer cleavage site [10]. This observation

Table 1 Binary patterns for nucleotides, A, U, C, G, and a loop/bulge structure, denoted by L, in PHDCleav [14] and LBSizeCleav with $k$ ones based on sequences and predicted secondary structures

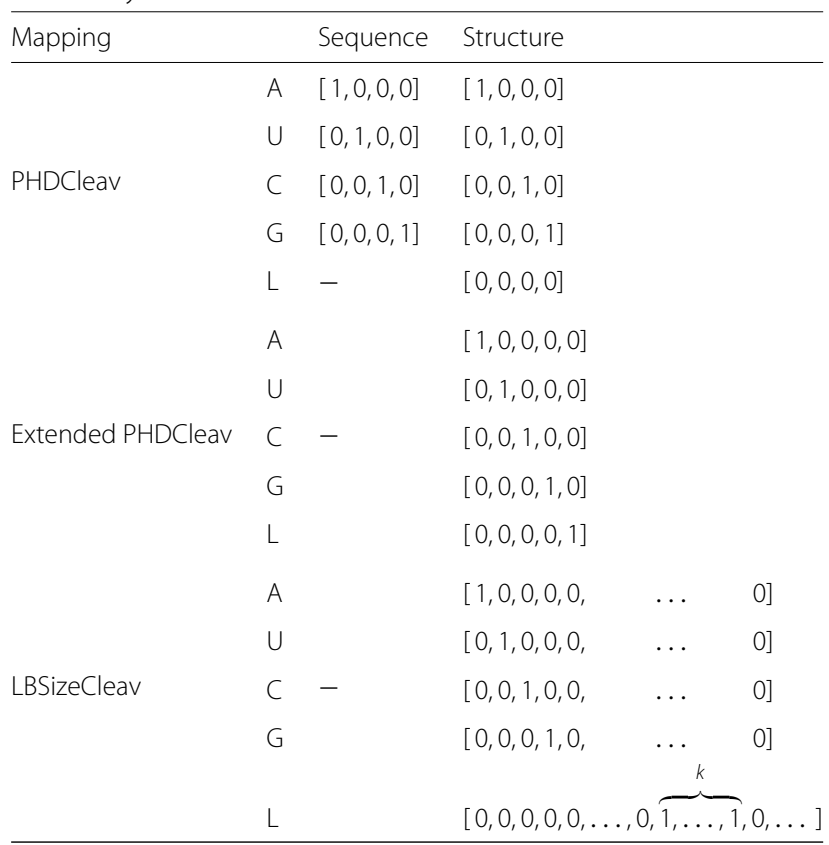

In PHDCleav binary patterns each nucleotide is represented by a 4-dimensional vector, and in PHDCleav Extended patterns each nucleotide is represented by a 5-dimensional vector, while in LBSizeCleav the dimension of the vector is $3+k+N$, in which $N$ denotes the maximum number of length of loop/bulges among all the pre-miRNAs in the training dataset 
suggests that the loop/bulge structure may be a feature that is useful for prediction of a Dicer cleavage site. The extended binary pattern of PHDCleav was developed on the basis of this assumption.

After obtaining the secondary structure from a given sequence by some prediction methods, in the extended binary pattern of PHDCleav, a nucleotide is converted to a five-dimensional vector as $[1,0,0,0,0],[0,1,0,0,0],[0$, $0,1,0,0],[0,0,0,1,0]$, and $[0,0,0,0,1]$, for A, U, C, G, and $L$, respectively, where $L$ indicates that the corresponding nucleotide is predicted to be in a loop/bulge structure. Just as the nucleotides in the window, its complementary nucleotides are also converted to a feature vector. After that, the dimensionality of the vector is $10 w$.

\section{Feature space mapping of LBSizeCleav}

It is reasonable to consider not only the position but also the length of loop/bulge structures. Therefore, we propose novel feature space mapping (LBSizeCleav) by introducing the length of a loop/bulge structure into the algorithm.

The binary pattern of LBSizeCleav is an extension of that of PHDCleav. Let $M$ be the maximal length of loops and bulges of all the pre-miRNAs in a dataset, and suppose $L_{l}$ indicates that the corresponding nucleotide is in a loop/bulge structure of length $l$. Here we introduce a new parameter named $k$ into LBSizeCleav, which is a positive integer representing the effect of length of loops and bulges to the kernel computation. Then, we designate a nucleotide without any loop/bulge structure for $k$ as a $(M+k+3)$-dimensional vector, namely, $[1,0,0,0, \ldots, 0]$, $[0,1,0,0, \ldots, 0],[0,0,1,0,0, \ldots, 0],[0,0,0,1,0, \ldots, 0]$ for A, U, C, and G, respectively (see Table 1). A nucleotide in a loop/bulge structure of length $l$ is represented as $[0, \ldots, 0,1, \ldots, 1,0, \ldots]$, where $k$ ones appear from the $(4+l)$-th element to the $(k+3+l)$-th element. Thus, for window size $w$, a $2 w(M+k+3)$-dimensional vector is generated.

Let $\boldsymbol{x}_{1}$ and $\boldsymbol{x}_{2}$ be binary patterns of $L_{l_{1}}$ and $L_{l_{2}}$, respectively. If we use the inner product for kernel computation, then the inner product between the binary patterns is $\boldsymbol{x}_{1}$. $\boldsymbol{x}_{2}=\max \left\{k-\left|l_{1}-l_{2}\right|, 0\right\}$. If we use the radial basis function (RBF) kernel, $\exp \left\{-\gamma\left\|\boldsymbol{x}_{1}-\boldsymbol{x}_{2}\right\|^{2}\right\}=\exp \left\{-4 \gamma \min \left\{\left(l_{1}-\right.\right.\right.$ $\left.\left.l_{2}\right)^{2}, k^{2}\right\}$, where $\gamma>0$. These values assume the maximum when $l_{1}=l_{2}$ and decrease according to the difference $\left|l_{1}-l_{2}\right|$ and $k$, while $\mathrm{k}$ gets larger, the value changes of kernel function is more sensitive to the size of $\left|l_{1}-l_{2}\right|$, in this way by controlling the value $\mathrm{k}$ we could control the sensitivity of our method to length of loops and bulges. Since PHDCleav used radial basis function (RBF), we also selected RBF as our kernel function.

Figure 1 illustrates the feature space mapping of LBSizeCleav for the pre-miRNA of the miRBase ID hsa-miR-200c with a predicted secondary structure, where nucleotides in the region removed by Dicer are shown as lowercase letters. CD-5p and CD-3p denote cleavage sites in 5p and 3p arms, respectively. Sequences in the red rectangles denote sequences used to generate feature vectors representing $5 p$ and $3 p$ arms, which are selected by the principle that the cleavage site is located at the center of the sequence. Here, we generate the feature vector of LBSizeCleav at $k=$ 3 and $w=6$ for the site CD-5p and for the site $6 \mathrm{nt}$ away from CD-5p. The nucleotides in the window in the $5 p$ arm are UGGgug, and loop/bulge structures are detected at two positions. As a result, $L_{1} \mathrm{GGg} L_{1} \mathrm{~g}$ is converted to

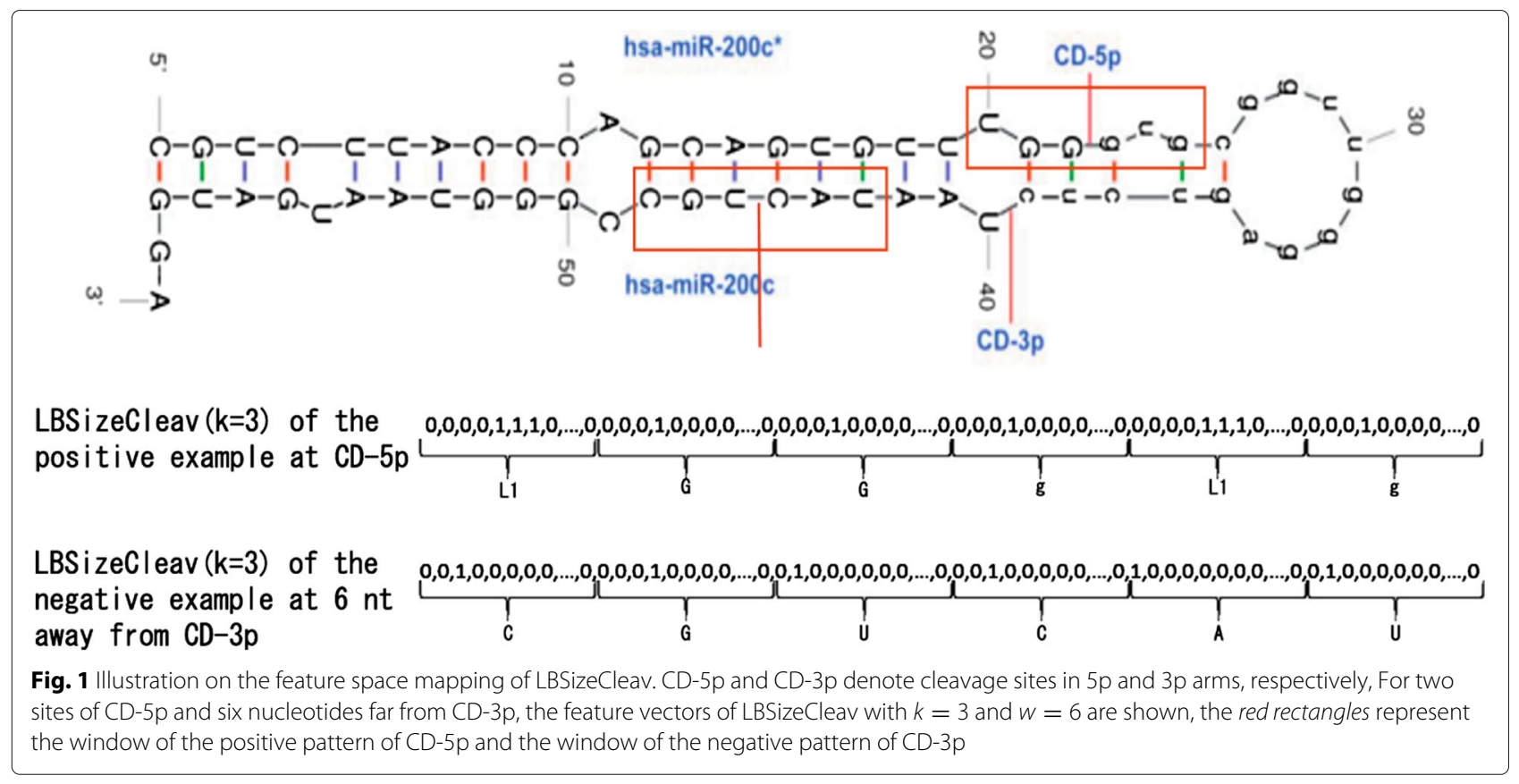


the $6(M+6)$-dimensional binary vector, where loop/bulge structures $L_{l}$ are inserted. For the 3p arm, CGUCAU is converted in accordance with Table 1.

\section{Results}

We retrieved 810 empirically validated sequences of premiRNAs from miRBase (version 21) [24], where cleavage sites CD-5p and CD-3p are both defined for each premiRNA. The pre-miRNAs were selected under the principle that both the cleavage sites of CD-5p and CD-3p are experimentally validated. (i.e. only precursors with cleavage sites at both CD-5p and CD-3p are selected, we made this choice to let our dataset be generated the in same way as dataset of PHDCleav)

Table 2 Results on average specificity, sensitivity, accuracy, and MCC for both $5 p$ and $3 p$ arms by five-fold cross-validation using PHDCleav and LBSizeCleav $(k=1, \cdots, 5)$ with window sizes $8,10,12,14$ based on sequences and secondary structures predicted by quikfold server

\begin{tabular}{|c|c|c|c|c|c|c|c|c|c|}
\hline \multirow{2}{*}{ Method } & \multirow{2}{*}{ Window size } & \multicolumn{4}{|c|}{$5 p$ arm } & \multicolumn{4}{|l|}{$3 p$ arm } \\
\hline & & $\mathrm{Sn}$ & $\mathrm{Sp}$ & $A C$ & $\mathrm{MCC}$ & $\mathrm{Sn}$ & Sp & $A C$ & $\mathrm{MCC}$ \\
\hline \multirow{4}{*}{ PHDCleav (sequence) } & 8 & 0.602 & 0.503 & 0.552 & 0.105 & 0.662 & 0.625 & 0.644 & 0.287 \\
\hline & 10 & 0.541 & 0.573 & 0.557 & 0.115 & 0.661 & 0.642 & 0.652 & 0.303 \\
\hline & 12 & 0.560 & 0.555 & 0.557 & 0.115 & 0.660 & 0.656 & 0.658 & 0.316 \\
\hline & 14 & 0.539 & 0.572 & 0.555 & 0.111 & 0.654 & 0.702 & 0.678 & 0.356 \\
\hline \multirow{4}{*}{ PHDCleav (structure) } & 8 & 0.753 & 0.814 & 0.784 & 0.568 & 0.670 & 0.661 & 0.665 & 0.330 \\
\hline & 10 & 0.784 & 0.827 & 0.806 & 0.612 & 0.702 & 0.719 & 0.710 & 0.421 \\
\hline & 12 & 0.790 & 0.842 & 0.816 & 0.633 & 0.739 & 0.764 & 0.752 & 0.503 \\
\hline & 14 & 0.799 & 0.857 & 0.828 & 0.657 & 0.779 & 0.783 & 0.781 & 0.562 \\
\hline \multirow{4}{*}{ Extended PHDCleav } & 8 & 0.750 & 0.798 & 0.774 & 0.548 & 0.652 & 0.716 & 0.684 & 0.369 \\
\hline & 10 & 0.779 & 0.827 & 0.803 & 0.607 & 0.674 & 0.783 & 0.729 & 0.460 \\
\hline & 12 & 0.809 & 0.845 & 0.827 & 0.654 & 0.714 & 0.790 & 0.752 & 0.506 \\
\hline & 14 & 0.813 & 0.868 & 0.840 & 0.682 & 0.781 & 0.801 & 0.791 & 0.582 \\
\hline \multirow{4}{*}{ LBSizeCleav $(k=1)$} & 8 & 0.668 & 0.924 & 0.796 & 0.612 & 0.630 & 0.684 & 0.657 & 0.315 \\
\hline & 10 & 0.709 & 0.947 & 0.828 & 0.675 & 0.651 & 0.776 & 0.713 & 0.430 \\
\hline & 12 & 0.774 & 0.945 & 0.859 & 0.730 & 0.686 & 0.847 & 0.766 & 0.540 \\
\hline & 14 & 0.808 & 0.933 & 0.871 & 0.747 & 0.758 & 0.874 & 0.816 & 0.637 \\
\hline \multirow{4}{*}{ LBSizeCleav $(k=2)$} & 8 & 0.662 & 0.954 & 0.808 & 0.645 & 0.626 & 0.723 & 0.674 & 0.351 \\
\hline & 10 & 0.725 & 0.946 & 0.835 & 0.688 & 0.642 & 0.806 & 0.724 & 0.455 \\
\hline & 12 & 0.784 & 0.938 & 0.861 & 0.731 & 0.665 & 0.882 & 0.773 & 0.560 \\
\hline & 14 & 0.820 & 0.925 & 0.872 & 0.749 & 0.734 & 0.916 & 0.825 & 0.661 \\
\hline \multirow{4}{*}{ LBSizeCleav $(k=3)$} & 8 & 0.692 & 0.949 & 0.821 & 0.664 & 0.619 & 0.735 & 0.677 & 0.356 \\
\hline & 10 & 0.752 & 0.941 & 0.846 & 0.706 & 0.618 & 0.822 & 0.720 & 0.450 \\
\hline & 12 & 0.803 & 0.932 & 0.867 & 0.741 & 0.635 & 0.914 & 0.774 & 0.571 \\
\hline & 14 & 0.825 & 0.912 & 0.869 & 0.740 & 0.719 & 0.942 & 0.830 & 0.678 \\
\hline \multirow{4}{*}{ LBSizeCleav $(k=4)$} & 8 & 0.695 & 0.949 & 0.822 & 0.667 & 0.614 & 0.736 & 0.675 & 0.353 \\
\hline & 10 & 0.767 & 0.938 & 0.853 & 0.716 & 0.621 & 0.835 & 0.728 & 0.467 \\
\hline & 12 & 0.815 & 0.927 & 0.871 & 0.747 & 0.639 & 0.912 & 0.776 & 0.573 \\
\hline & 14 & 0.835 & 0.909 & 0.872 & 0.746 & 0.723 & 0.924 & 0.823 & 0.660 \\
\hline \multirow{4}{*}{ LBSizeCleav $(k=5)$} & 8 & 0.700 & 0.947 & 0.824 & 0.668 & 0.594 & 0.771 & 0.682 & 0.371 \\
\hline & 10 & 0.772 & 0.936 & 0.854 & 0.717 & 0.578 & 0.862 & 0.720 & 0.459 \\
\hline & 12 & 0.821 & 0.924 & 0.872 & 0.749 & 0.634 & 0.921 & 0.777 & 0.579 \\
\hline & 14 & 0.838 & 0.909 & 0.874 & 0.749 & 0.724 & 0.932 & 0.828 & 0.671 \\
\hline
\end{tabular}

$\mathrm{Sn}, \mathrm{Sp}, \mathrm{Ac}$, and MCC denote sensitivity, specificity, accuracy, and Matthews correlation coefficient, respectively 
All the pre-miRNAs are selected from human premiRNAs.

We used these cleavage sites as positive examples using windows of size $8,10,12,14 \mathrm{nt}$, where each window was selected so that a cleavage site is located at the center of the window, and we generated negative examples on the same sequence so that both centers of the positive and negative examples were $6 \mathrm{nt}$ away from each other, as in the previous study [14]. This approach is based on the assumption that for most pre-miRNAs, the Dicer cleavage site is seldom selected at the center of the hairpin structure. In PHDCleav, two secondary structure predictors, quikfold [25] and RNAFold from ViennaRNA [26] were used, hence, we used both the RNAFold from ViennaRNA. and the quikfold server (version 3.0, http://mfold.rna. albany.edu/?q=DINAMelt/Quickfold) for prediction of RNA secondary structures. The results were given in Tables 2 and 3. Because in PHDCleav, the accuracy of prediction by nucleotide composition was worse than that by binary patterns, we compared our method with the binary patterns of PHDCleav. We used the libSVM 3.18 package [27] with the RBF kernel to utilize SVM because the RBF kernel was used in PHDCleav.

The performance of prediction methods was assessed by means of sensitivity, specificity, accuracy, and the Matthews correlation coefficient (MCC), defined as follows:

$$
\begin{aligned}
\text { sensitivity } & =\frac{T P}{T P+F N} \\
\text { specificity } & =\frac{T N}{T N+F P}, \\
\text { accuracy } & =\frac{T P+T N}{T P+F P+T N+F N} \\
M C C & =\frac{T P \cdot T N-F P \cdot F N}{\sqrt{(T P+F P)(T P+F N)(T N+F P)(T N+F N)}}
\end{aligned}
$$

\begin{tabular}{|c|c|c|c|c|c|c|c|c|c|}
\hline \multirow{2}{*}{ Method } & \multirow{2}{*}{ Window size } & \multicolumn{4}{|c|}{$5 p$ arm } & \multicolumn{4}{|l|}{$3 p$ arm } \\
\hline & & Sn & $\mathrm{Sp}$ & $A C$ & MCC & Sn & $\mathrm{Sp}$ & $A C$ & MCC \\
\hline \multirow{4}{*}{ Extended PHDCleav } & 8 & 0.746 & 0.744 & 0.745 & 0.490 & 0.772 & 0.750 & 0.761 & 0.522 \\
\hline & 10 & 0.792 & 0.783 & 0.787 & 0.575 & 0.779 & 0.800 & 0.790 & 0.580 \\
\hline & 12 & 0.798 & 0.799 & 0.798 & 0.597 & 0.785 & 0.830 & 0.808 & 0.616 \\
\hline & 14 & 0.778 & 0.813 & 0.795 & 0.591 & 0.805 & 0.853 & 0.829 & 0.659 \\
\hline \multirow{4}{*}{ LBSizeCleav $(k=1)$} & 8 & 0.739 & 0.805 & 0.772 & 0.545 & 0.785 & 0.790 & 0.788 & 0.576 \\
\hline & 10 & 0.798 & 0.820 & 0.809 & 0.618 & 0.795 & 0.815 & 0.805 & 0.610 \\
\hline & 12 & 0.792 & 0.815 & 0.803 & 0.607 & 0.822 & 0.840 & 0.831 & 0.662 \\
\hline & 14 & 0.815 & 0.822 & 0.819 & 0.638 & 0.851 & 0.852 & 0.851 & 0.703 \\
\hline \multirow{4}{*}{ LBSizeCleav $(k=2)$} & 8 & 0.753 & 0.788 & 0.771 & 0.542 & 0.792 & 0.788 & 0.790 & 0.580 \\
\hline & 10 & 0.816 & 0.795 & 0.806 & 0.612 & 0.811 & 0.794 & 0.803 & 0.606 \\
\hline & 12 & 0.836 & 0.784 & 0.810 & 0.621 & 0.814 & 0.803 & 0.808 & 0.617 \\
\hline & 14 & 0.845 & 0.769 & 0.807 & 0.616 & 0.867 & 0.800 & 0.834 & 0.669 \\
\hline \multirow{4}{*}{ LBSizeCleav $(k=3)$} & 8 & 0.751 & 0.794 & 0.773 & 0.546 & 0.784 & 0.797 & 0.790 & 0.581 \\
\hline & 10 & 0.808 & 0.808 & 0.808 & 0.615 & 0.795 & 0.813 & 0.804 & 0.608 \\
\hline & 12 & 0.822 & 0.800 & 0.811 & 0.623 & 0.808 & 0.835 & 0.821 & 0.643 \\
\hline & 14 & 0.816 & 0.803 & 0.809 & 0.619 & 0.853 & 0.838 & 0.846 & 0.692 \\
\hline \multirow{4}{*}{ LBSizeCleav $(k=4)$} & 8 & 0.764 & 0.772 & 0.768 & 0.536 & 0.809 & 0.772 & 0.790 & 0.581 \\
\hline & 10 & 0.824 & 0.762 & 0.793 & 0.587 & 0.824 & 0.766 & 0.795 & 0.590 \\
\hline & 12 & 0.841 & 0.737 & 0.789 & 0.581 & 0.842 & 0.756 & 0.799 & 0.600 \\
\hline & 14 & 0.871 & 0.678 & 0.774 & 0.559 & 0.898 & 0.697 & 0.797 & 0.607 \\
\hline \multirow{4}{*}{ LBSizeCleav $(k=5)$} & 8 & 0.782 & 0.747 & 0.764 & 0.529 & 0.822 & 0.744 & 0.783 & 0.568 \\
\hline & 10 & 0.836 & 0.732 & 0.784 & 0.572 & 0.829 & 0.726 & 0.777 & 0.558 \\
\hline & 12 & 0.867 & 0.699 & 0.783 & 0.574 & 0.864 & 0.682 & 0.773 & 0.556 \\
\hline & 14 & 0.899 & 0.626 & 0.763 & 0.546 & 0.917 & 0.619 & 0.768 & 0.562 \\
\hline
\end{tabular}

Table 3 Results on average specificity, sensitivity, accuracy, and MCC for both $5 p$ and $3 p$ arms by five-fold cross-validation using PHDCleav and LBSizeCleav $(k=1, \cdots, 5)$ with window sizes $8,10,12,14$ based on secondary structures predicted by RNAFold

$\mathrm{Sn}, \mathrm{Sp}, \mathrm{Ac}$, and MCC denote sensitivity, specificity, accuracy, and Matthews correlation coefficient, respectively 
where $T P, T N, F P, F N$ denote the number of true positive, true negative, false positive, and false negative results, respectively.

We performed fivefold cross-validation, and used the average sensitivity, specificity, accuracy, and MCC. We examined size $w$ of a window from 8 to 14 and the number $k$ of ones in LBSizeCleav from 1 to 5 for $5 p$ and $3 p$ arms of pre-miRNAs. Table 2 shows the results of PHDCleav and LBSizeCleav $(k=1, \cdots, 5)$ based on sequences and secondary structures predicted by the quikfold server. In terms of prediction performance in the $5 \mathrm{p}$ arm of premiRNA, the best result of PHDCleav corresponded to the accuracy of $84.0 \%$, whereas LBSizeCleav at $k=5$ achieved the accuracy of $87.4 \%$. In addition, the values

Table 4 Variances of specificity, sensitivity, accuracy, and MCC for both $5 p$ and $3 p$ arms by five-fold cross-validation using PHDCleav and LBSizeCleav $(k=1, \cdots, 5)$ with window sizes $8,10,12,14$ based on sequences and secondary structures predicted by quikfold server

\begin{tabular}{|c|c|c|c|c|c|c|c|c|c|}
\hline \multirow{2}{*}{ feature extraction method } & \multirow{2}{*}{ Window size } & \multicolumn{4}{|l|}{$C D-5 p$} & \multicolumn{4}{|l|}{$C D-3 p$} \\
\hline & & Sn & Sp & $A c$ & $M c$ & Sn & $\mathrm{Sp}$ & $A C$ & $M c$ \\
\hline \multirow{4}{*}{ PHDCleav (sequence) } & 8 & 0.0137 & 0.0008 & 0.0074 & 0.0036 & 0.0072 & 0.0015 & 0.0094 & 0.0066 \\
\hline & 10 & 0.0184 & 0.0004 & 0.0111 & 0.0018 & 0.0044 & 0.0005 & 0.0072 & 0.0024 \\
\hline & 12 & 0.0208 & 0.0001 & 0.0223 & 0.0003 & 0.0078 & 0.0011 & 0.0031 & 0.0046 \\
\hline & 14 & 0.0293 & 0.0009 & 0.0174 & 0.0037 & 0.0065 & 0.0007 & 0.0048 & 0.0029 \\
\hline \multirow{4}{*}{ PHDCleav (structure) } & 8 & 0.0042 & 0.0039 & 0.0067 & 0.0155 & 0.0187 & 0.0013 & 0.0091 & 0.0062 \\
\hline & 10 & 0.0026 & 0.0043 & 0.0088 & 0.0177 & 0.0100 & 0.0014 & 0.0050 & 0.0059 \\
\hline & 12 & 0.0042 & 0.0027 & 0.0034 & 0.0109 & 0.0051 & 0.0011 & 0.0024 & 0.0045 \\
\hline & 14 & 0.0047 & 0.0031 & 0.0034 & 0.0125 & 0.0039 & 0.0012 & 0.0014 & 0.0047 \\
\hline \multirow{4}{*}{ Extended PHDCleav } & 8 & 0.0029 & 0.0032 & 0.0063 & 0.0128 & 0.0123 & 0.0025 & 0.0043 & 0.0103 \\
\hline & 10 & 0.0030 & 0.0038 & 0.0061 & 0.0154 & 0.0064 & 0.0019 & 0.0016 & 0.0075 \\
\hline & 12 & 0.0040 & 0.0033 & 0.0050 & 0.0136 & 0.0054 & 0.0015 & 0.0011 & 0.0059 \\
\hline & 14 & 0.0059 & 0.0027 & 0.0016 & 0.0108 & 0.0032 & 0.0013 & 0.0010 & 0.0052 \\
\hline \multirow{4}{*}{ LBSizeCleav $(k=1)$} & 8 & 0.0030 & 0.0025 & 0.0044 & 0.0115 & 0.0100 & 0.0004 & 0.0074 & 0.0019 \\
\hline & 10 & 0.0022 & 0.0015 & 0.0013 & 0.0064 & 0.0078 & 0.0011 & 0.0015 & 0.0042 \\
\hline & 12 & 0.0050 & 0.0024 & 0.0010 & 0.0090 & 0.0077 & 0.0015 & 0.0002 & 0.0051 \\
\hline & 14 & 0.0075 & 0.0035 & 0.0010 & 0.0132 & 0.0036 & 0.0007 & 0.0002 & 0.0026 \\
\hline \multirow{4}{*}{ LBSizeCleav $(k=2)$} & 8 & 0.0042 & 0.0018 & 0.0008 & 0.0066 & 0.0053 & 0.0010 & 0.0036 & 0.0041 \\
\hline & 10 & 0.0038 & 0.0020 & 0.0009 & 0.0076 & 0.0034 & 0.0010 & 0.0010 & 0.0039 \\
\hline & 12 & 0.0051 & 0.0029 & 0.0016 & 0.0115 & 0.0042 & 0.0017 & 0.0008 & 0.0063 \\
\hline & 14 & 0.0051 & 0.0028 & 0.0012 & 0.0107 & 0.0043 & 0.0008 & 0.0002 & 0.0024 \\
\hline \multirow{4}{*}{ LBSizeCleav $(k=3)$} & 8 & 0.0025 & 0.0013 & 0.0008 & 0.0050 & 0.0070 & 0.0010 & 0.0021 & 0.0042 \\
\hline & 10 & 0.0039 & 0.0022 & 0.0012 & 0.0086 & 0.0064 & 0.0013 & 0.0006 & 0.0048 \\
\hline & 12 & 0.0063 & 0.0031 & 0.0012 & 0.0119 & 0.0039 & 0.0015 & 0.0005 & 0.0055 \\
\hline & 14 & 0.0060 & 0.0033 & 0.0015 & 0.0130 & 0.0073 & 0.0016 & 0.0003 & 0.0046 \\
\hline \multirow{4}{*}{ LBSizeCleav $(k=4)$} & 8 & 0.0029 & 0.0016 & 0.0009 & 0.0064 & 0.0066 & 0.0020 & 0.0030 & 0.0078 \\
\hline & 10 & 0.0046 & 0.0025 & 0.0011 & 0.0095 & 0.0071 & 0.0014 & 0.0006 & 0.0049 \\
\hline & 12 & 0.0061 & 0.0032 & 0.0014 & 0.0124 & 0.0033 & 0.0011 & 0.0007 & 0.0041 \\
\hline & 14 & 0.0051 & 0.0030 & 0.0015 & 0.0120 & 0.0088 & 0.0025 & 0.0002 & 0.0082 \\
\hline \multirow{4}{*}{ LBSizeCleav $(k=5)$} & 8 & 0.0029 & 0.0017 & 0.0009 & 0.0066 & 0.0062 & 0.0021 & 0.0031 & 0.0082 \\
\hline & 10 & 0.0055 & 0.0029 & 0.0013 & 0.0113 & 0.0032 & 0.0011 & 0.0005 & 0.0042 \\
\hline & 12 & 0.0051 & 0.0029 & 0.0015 & 0.0114 & 0.0029 & 0.0011 & 0.0009 & 0.0044 \\
\hline & 14 & 0.0047 & 0.0029 & 0.0016 & 0.0116 & 0.0076 & 0.0023 & 0.0002 & 0.0076 \\
\hline
\end{tabular}

Sn, Sp, Ac, and MCC denote sensitivity, specificity, accuracy, and Matthews correlation coefficient, respectively 


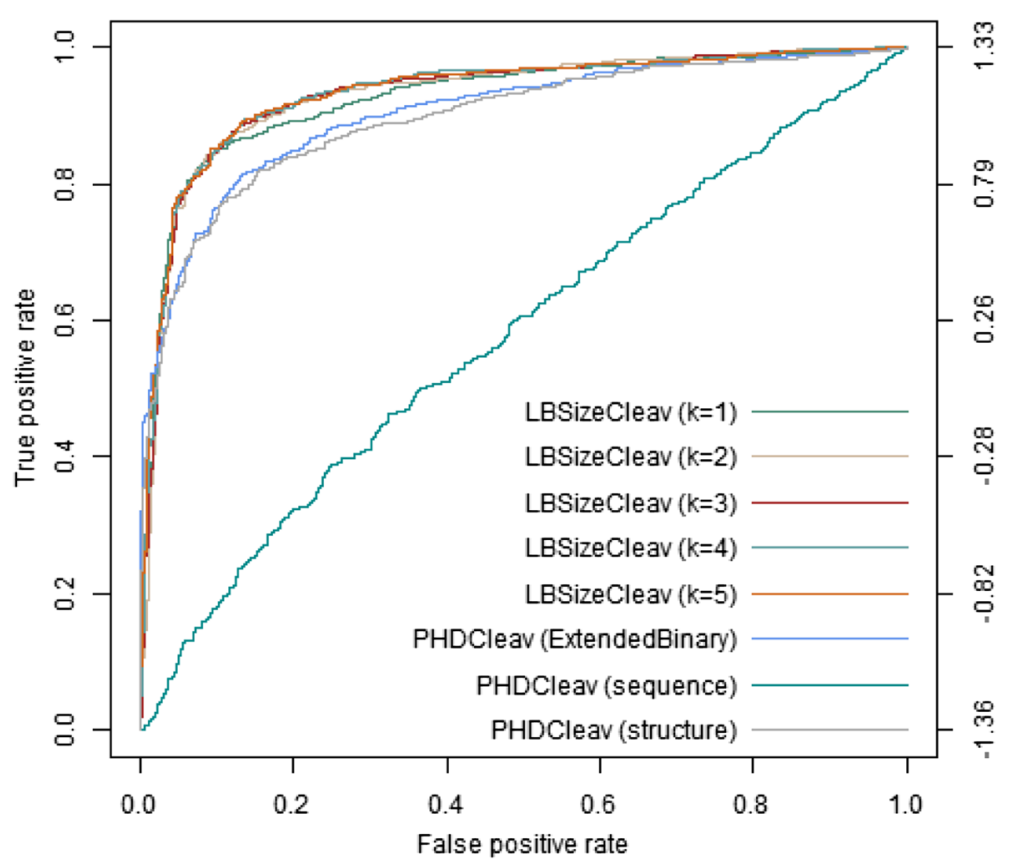

Fig. 2 Results on ROC curves by LBSizeCleav and PHDCleav with window size $w=14$ for $5 p$ arm. From the figure we could see that the ROC curve of LBSizeCleav from $k=1$ to $k=5$ is significantly better than binary Pattern and extended binary pattern of PHDCleav for both $5 p$ and $3 p$ arms

of prediction accuracy of LBSizeCleav at $w=12,14$ were higher than those of PHDCleav. As for prediction performance in the $3 \mathrm{p}$ arm of pre-miRNA, the best result of PHDCleav corresponded to the accuracy of $79.1 \%$, whereas LBSizeCleav achieved the accuracy of $83.0 \%$.
Table 3 shows the results of PHDCleav and LBSizeCleav $(k=1, \cdots, 5)$ based on sequences and secondary structures predicted by the RNAFold. In terms of prediction performance in the $5 \mathrm{p}$ arm of pre-miRNA, the best result of PHDCleav corresponded to the accuracy of $81.3 \%$,

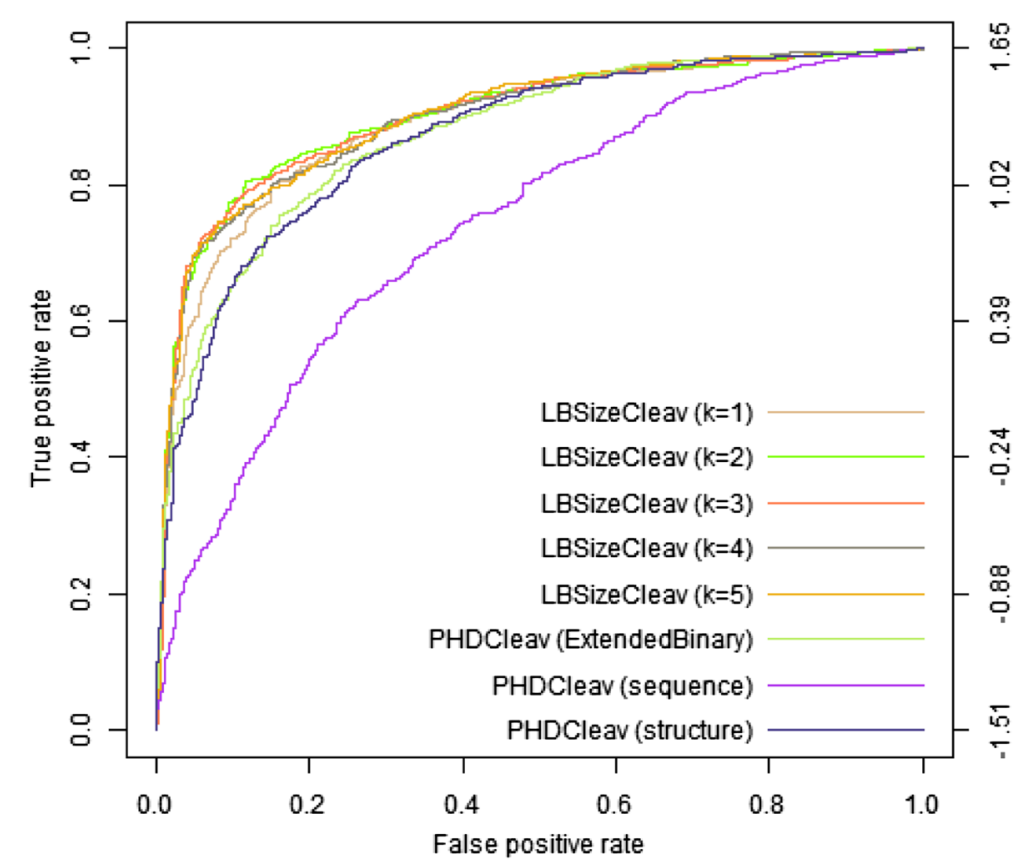

Fig. 3 Results on ROC curves by LBSizeCleav and PHDCleav with window size $w=14$ for $3 p$ arm. From the figure we could see that the ROC curve of LBSizeCleav from $k=1$ to $k=5$ is significantly better than binary Pattern and extended binary pattern of PHDCleav for both $5 p$ and $3 p$ arms 


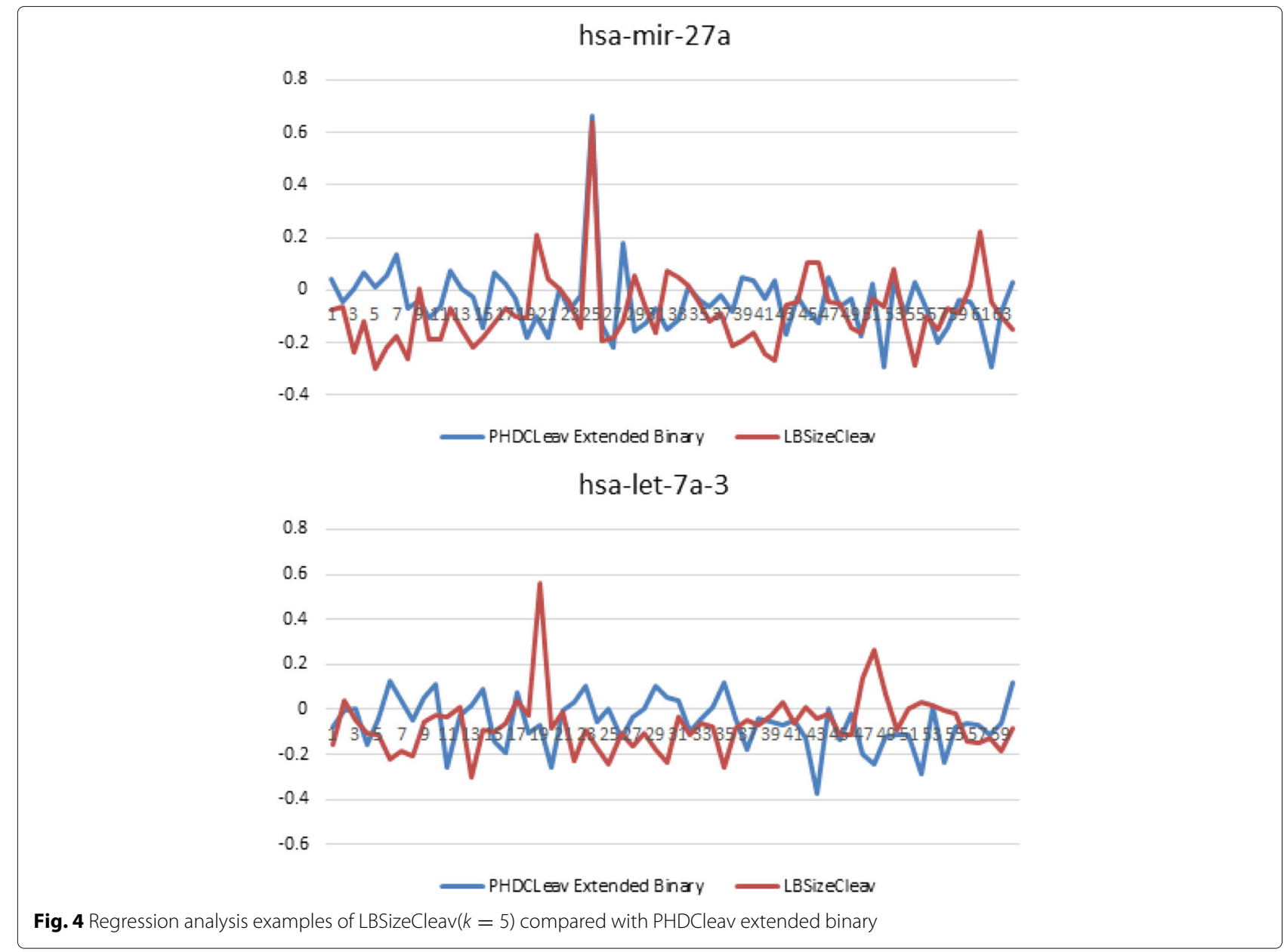

whereas LBSizeCleav at $k=1$ achieved the accuracy of $82.2 \%$. As for prediction performance in the $3 p$ arm of pre-miRNA, the best result of PHDCleav corresponded to the accuracy of $82.9 \%$, whereas LBSizeCleav achieved the accuracy of $85.1 \%$.
To better evaluate the performance we also calculated the variance of each prediction result in Table 4. Figures 2 and 3 show the results of LBSizeCleav and PHDCleav on receiver-operator characteristic (ROC) curves at window size $w=14$ in $5 \mathrm{p}$ and $3 \mathrm{p}$ arms. Judging by the

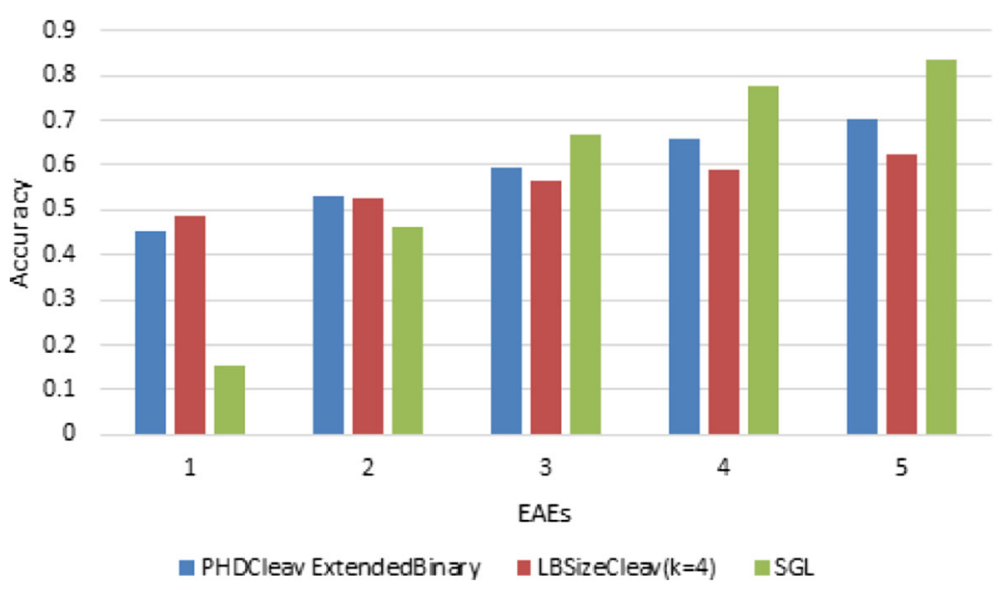

Fig. 5 Result on accuracy of LBSizeCleav $(k=5)$ compared with PHDCleav extended binary and SGL of prediction in CD-5p 
performance evaluation, our newly developed method outperformed the binary patterns of PHDCleav; this finding was suggestive of efficiency of the feature representing the length of loop/bulge structures.

Since in our results, LBSizeCleav with parameters of $w=14, k=5$ outperforms the others, we selected these parameters as our parameters for prediction model. For an input sequence, we created a shift window of size 14 nt shifting from the $5 p$ arm to the $3 p$ arm. For each shift window we performed an SVM regression analysis using our model. Here we randomly selected 2 precursors from the dataset and showed the score of the extended binary pattern of PHDCleav and LBSizeCleav with $k=5$. From the result we could see that although both tools have predicted the cleavage site correctly, LBSizeCleav predicted more true negatives than extended binary pattern of PHDCleav, which indicates a better performance in identifying negative patterns of LBSizeCleav (see in Fig. 4).

We also compared the performance of our tools with another state-of-art method, a recent published paper introduced an easy way named SGL (Simple Geometric Locater) to calculate the cleavage site of miRNA which
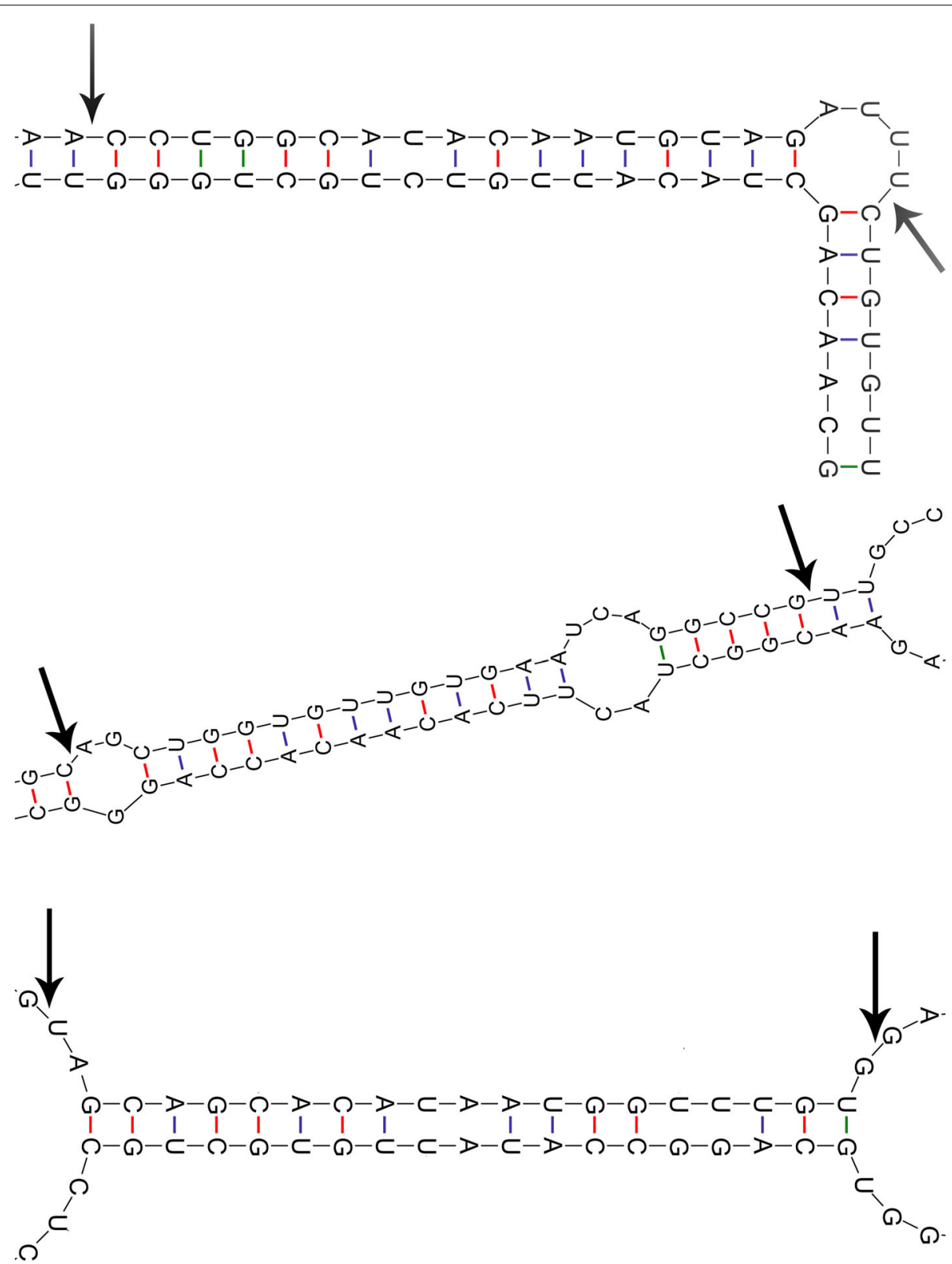

Fig. 6 Secondary structures of hsa-mir-221, hsa-mir-138-1, hsr-mir-15a predicted by quikfold server. The black arrow means the cleavage site validated by biological experiments 
Table 5 Number of patterns predicted only by LBSizeCleav $(k=1,4) /$ PHDCleav(extended binary) using secondary structure predicted by quikfold

\begin{tabular}{|c|c|c|c|}
\hline & & $5^{\prime}$-arm & 3'-arm \\
\hline \multirow{2}{*}{ Positive } & Only predicted by LBSizeCleav $(k=1)$ compared with PHDCleav (extended binary) & 39 & 39 \\
\hline & Only predicted by PHDCleav (extended binary) compared with LBSizeCleav $(k=1)$ & 57 & 38 \\
\hline \multirow{2}{*}{ Negative } & Only predicted by LBSizeCleav $(k=1)$ compared with PHDCleav (extended binary) & 82 & 65 \\
\hline & Only predicted by PHDCleav (extended binary) compared with LBSizeCleav $(k=1)$ & 23 & 12 \\
\hline \multirow{2}{*}{ Positive } & Only predicted by LBSizeCleav $(k=4)$ compared with PHDCleav(extended binary) & 39 & 39 \\
\hline & Only predicted by PHDCleav compared with LBSizeCleav $(k=4)$ & 57 & 38 \\
\hline \multirow{2}{*}{ Negative } & Only predicted by LBSizeCleav $(k=4)$ compared with PHDCleav(extended binary) & 82 & 65 \\
\hline & Only predicted by PHDCleav (extended binary) compared with LBSizeCleav $(k=4)$ & 23 & 12 \\
\hline
\end{tabular}

outperforms other methods. We generated a benchmark to compare our method as well as PHDCleav with SGL of prediction in CD-5p, which result is shown in Fig. 5. In this benchmark we selected the threshold (0.0) of LBSizeCleav as well as PHDCleav and calculated the EAEs(End Absolute Error, the absolute error of the predicted minus the true position for a specific duplex end) from the true cleavage site and compared it with the SGL method. From the result we could see that although at high EAEs PHDCleav outperforms LBSizeCleav, LBSizeCleav outperforms both PHDCleav and SGL at EAE 1, which indicates that LBSizeCleav predicted less false positives than PHDCleav.

\section{Discussion}

There were several pre-miRNAs, such as pre-mir221, pre-mir138-1, and pre-mir-15a, that were identified by LBSizeCleav but were not identified by PHDCleav in the prediction results from the $5 \mathrm{p}$ arm of a pre-miRNA with a shift window of 14 nt (see Fig. 6). By comparing these three pre-miRNAs, we found that all of them contain a part of loop/bulge structures that is more than $1 \mathrm{nt}$ long in their mature parts. This result indicates that the length of a loop/bulge structure is an important determinant of a cleavage site. Careful analysis revealed that pre-mir221 and pre-mir138-1 contain their loop/bulge structures in their bulge parts, whereas pre-mir-15a has its loop/bulge structure in its loop part, proving that both loop and bulge structures can affect the cleavage site selection. To further evaluate the effect of the length of a loop/bulge structure in affecting the cleavage site selection we calculated the number of pre-miRNAs which LBSizeCleav identified successfully while PHDCleav failed to identify and vice versa (see Table 5). From the result we could see that for the positive patterns our method performs almost the same as PHDCleav, but for negative patterns our method shows a significant improvement in accuracy. This result indicates that our method has a better resolution in identifying negative patterns.

\section{Conclusions}

In this study, we proposed a novel methodLBSizeCleav-for prediction of Dicer cleavage sites. By integrating information on the length of a loop/bulge structure of a pre-miRNA (as predicted by the quikfold server), we developed novel feature space mapping. We performed fivefold cross-validation for validated pre-miRNA sequences from miRBase. In both $5 p$ and $3 p$ arms, the proposed method showed better performance than did the binary patterns of PHDCleav. This study shows a new way of feature evaluation; moreover, the better performance of our method points to the effectiveness of analysis of loop/bulge length at detecting Dicer cleavage sites.

\section{Abbreviations}

DGCR8: DiGeorge syndrome chromosomal region 8; dsRND: Double-stranded RNA-binding domain; FN: false negative; FP: False positive; miRNA: MicroRNA; PAZ: PIWI, AGO, and Zwille domain; RISC: RNA-induced silencing complex; SVM: Support Vector Machine; TN: True negative; TP: True positive

\section{Acknowledgments}

None.

\section{Funding}

TA was partially supported by Grant-in-Aid \# 26240034 from the Japan Society for Promotion of Science (JSPS); MH was in part supported by Grant-in-Aid \# 24500361 from JSPS.

Availability of data and materials

Related data and source code is avaliable at http://sunflower.kuicr.kyoto-u.ac. .jp/ houu/LBSizeCleav/index.html.

\section{Authors' contributions}

YB collected data and implemented the algorithm. MH helped implementation and computational experiment. YB and TA developed the algorithm. TA, MH supervised the whole project. YB carried out data interpretation and finalized the writing. All authors read and approved the final manuscript.

\section{Competing interests}

The authors declare that they have no competing interests.

\section{Consent for publication}

Not applicable.

Ethics approval and consent to participate

Not applicable. 
Received: 11 April 2016 Accepted: 11 November 2016

Published online: 25 November 2016

\section{References}

1. Bartel DP. MicroRNAs: genomics, biogenesis, mechanism, and function. Cell. 2004;116(2):281-97.

2. Bernstein E, Caudy AA, Hammond SM, Hannon GJ. Role for a bidentate ribonuclease in the initiation step of RNA interference. Nature. 2001;409(6818):363-6.

3. Lee $Y$, Jeon K, Lee JT, Kim S, Kim VN. MicroRNA maturation: stepwise processing and subcellular localization. EMBO J. 2002;21(17):4663-670.

4. Zamore PD, Tuschl T, Sharp PA, Bartel DP. RNAi: double-stranded RNA directs the ATP-dependent cleavage of mRNA at 21 to 23 nucleotide intervals. Cell. 2000;101(1):25-33.

5. Elbashir SM, Harborth J, Lendeckel W, Yalcin A, Weber K, Tuschl T. Duplexes of 21-nucleotide RNAs mediate RNA interference in cultured mammalian cells. Nature. 2001;411(6836):494-8.

6. Hammond SM, Bernstein E, Beach D, Hannon GJ. An RNA-directed nuclease mediates post-transcriptional gene silencing in Drosophila cells. Nature. 2000;404(6775):293-6.

7. MacRae IJ, Zhou K, Li F, Repic A, Brooks AN, Cande WZ, Adams PD, Doudna JA. Structural basis for double-stranded RNA processing by Dicer. Science. 2006;311(5758):195-8.

8. Lau PW, Guiley KZ, De N, Potter CS, Carragher B, MacRae IJ. The molecular architecture of human Dicer. Nat Struct Mol Biol. 2012;19(4): 436-40.

9. Park JE, Heo I, Tian Y, Simanshu DK, Chang H, Jee D, Patel DJ, Kim VN. Dicer recognizes the 5 [prime] end of RNA for efficient and accurate processing. Nature. 2011;475(7355):201-5.

10. Gu S, Jin L, Zhang Y, Huang Y, Zhang F, Valdmanis PN, Kay MA. The loop position of shRNAs and pre-miRNAs is critical for the accuracy of dicer processing in vivo. Cell. 2012;151(4):900-11.

11. Feng $Y$, Zhang X, Graves $P$, Zeng $Y$. A comprehensive analysis of precursor microRNA cleavage by human Dicer. RNA. 2012;18(11): 2083-092.

12. MacRae IJ, Zhou K, Doudna JA. Structural determinants of RNA recognition and cleavage by Dicer. Nat Struct Mol Biol. 2007;14(10): 934-40.

13. Jin $Y$, Lee CG. Single nucleotide polymorphisms associated with microRNA regulation. Biomolecules. 2013;3(2):287-302.

14. Ahmed F, Kaundal R, Raghava GP. PHDcleav: a SVM based method for predicting human Dicer cleavage sites using sequence and secondary structure of miRNA precursors. BMC Bioinforma. 2013;14(Suppl 14):9.

15. Drucker H, Wu D, Vapnik VN. Support vector machines for spam categorization. IEEE Trans Neural Netw. 1999;10(5):1048-1054.

16. Bhasin M, Raghava G. ESLpred: SVM-based method for subcellular localization of eukaryotic proteins using dipeptide composition and PSI-BLAST. Nucleic Acids Res. 2004;32(suppl 2):414-9.

17. Cai C, Han L, Ji ZL, Chen X, Chen YZ. SVM-Prot: web-based support vector machine software for functional classification of a protein from its primary sequence. Nucleic Acids Res. 2003;31(13):3692-697.

18. Zavaljevski N, Stevens FJ, Reifman J. Support vector machines with selective kernel scaling for protein classification and identification of key amino acid positions. Bioinformatics. 2002;18(5):689-96.

19. Kumar M, Gromiha MM, Raghava G. Prediction of RNA binding sites in a protein using SVM and PSSM profile. Proteins Struct Funct Bioinforma. 2008;71(1):189-94.

20. Ng KLS, Mishra SK. De novo SVM classification of precursor microRNAs from genomic pseudo hairpins using global and intrinsic folding measures. Bioinformatics. 2007;23(11):1321-1330.

21. Burbidge R, Trotter M, Buxton B, Holden S. Drug design by machine learning: support vector machines for pharmaceutical data analysis. Comput Chem. 2001;26(1):5-14.

22. Byvatov E, Fechner U, Sadowski J, Schneider G. Comparison of support vector machine and artificial neural network systems for drug/nondrug classification. J Chem Inf Comput Sci. 2003;43(6):1882-1889.

23. Lyngs $\varnothing \mathrm{RB}$, Zuker M, Pedersen CN. Internal loops in RNA secondary structure prediction. In: Proceedings of the Third Annual International Conference on Computational Molecular Biology. New York: ACM; 1999. p. 260-7.
24. Griffiths-Jones S, Grocock RJ, Van Dongen S, Bateman A, Enright AJ. miRBase: microRNA sequences, targets and gene nomenclature. Nucleic Acids Res. 2006;34(suppl 1):140-4.

25. Markham NR, Zuker M. UNAFold. In: Bioinformatics. Springer; 2008. http://link.springer.com/protocol/10.1007/978-1-60327-429-6_1.

26. Hofacker IL. Vienna RNA secondary structure server. Nucleic Acids Res. 2003;31(13):3429-431.

27. Chang CC, Lin CJ. LIBSVM: A library for support vector machines. ACM Trans Intell Syst Technol (TIST). 2011;2(3):27.

\section{Submit your next manuscript to BioMed Central and we will help you at every step:}

- We accept pre-submission inquiries

- Our selector tool helps you to find the most relevant journal

- We provide round the clock customer support

- Convenient online submission

- Thorough peer review

- Inclusion in PubMed and all major indexing services

- Maximum visibility for your research

Submit your manuscript at www.biomedcentral.com/submit
C Biomed Central 